\title{
In situ Four Dimensional (4D) X-ray Microtomography of the Compressive Behavior of eTPU Foam for High Performance Footware
}

\author{
A.S. Singaravelu ${ }^{1}$, Jason J. Williams ${ }^{1}$, Jasmin Walter ${ }^{2}$, Chris Holmes ${ }^{2}$, Mark Henderson ${ }^{2}$, and \\ Nikhilesh Chawla, ${ }^{1, *}$ \\ 1. Center for 4D Materials Science, Materials Science and Engineering, Arizona State University, \\ Tempe, AZ, USA. \\ 2. Future Team, Adidas, Nürnberg, Germany. \\ * Corresponding author: nchawla@asu.edu
}

In 2013 Adidas launched an industry changing innovation in the form of BOOST ${ }^{\mathrm{TM}}$, which is a novel footwear cushioning material. A midsole is generated from $~ 3000$ individual hollow particles (eTPU) to make a high performance foam with unique stiffness, viscoelasticity, and resistance to low temperatures. The unique properties of eTPU particles are known to be dominated by the TPU polymer used and cell morphology created during the foaming process. The cell morphology, distribution, and structural effects on mechanical properties need to be investigated to develop the next generation of high performance cushioning materials. Without a greater understanding of how a particle's cellular structure influences the mechanical properties during compression, future optimization of the material performance will rely on the existing experimental approach which is both time and resource intensive.

We have investigated the structure of individual beads as well as the foam (hundreds of beads fused together) using 4D x-ray microtomography. The behavior of the foam under real-time compression loading was also investigated. The primary goal for the project is to characterize the behavior of these samples during compression. Figure 1 shows in the in situ compression device for applying strain to the individual beads. Figure 2 shows virtual slices at 0 and $50 \%$ compressive strain. Note that the bead exhibits a heterogeneous distribution of cell sizes, ranging from larger in the center to smaller near the surface. During compression, intense densification takes place near the compressive plattens. Localized buckling of the cells takes place, particularly where the ligaments are relatively thin. By characterizing the changes in the materials cellular structure during compression, we are able to understand how their cell morphology influences the mechanical properties. 
A

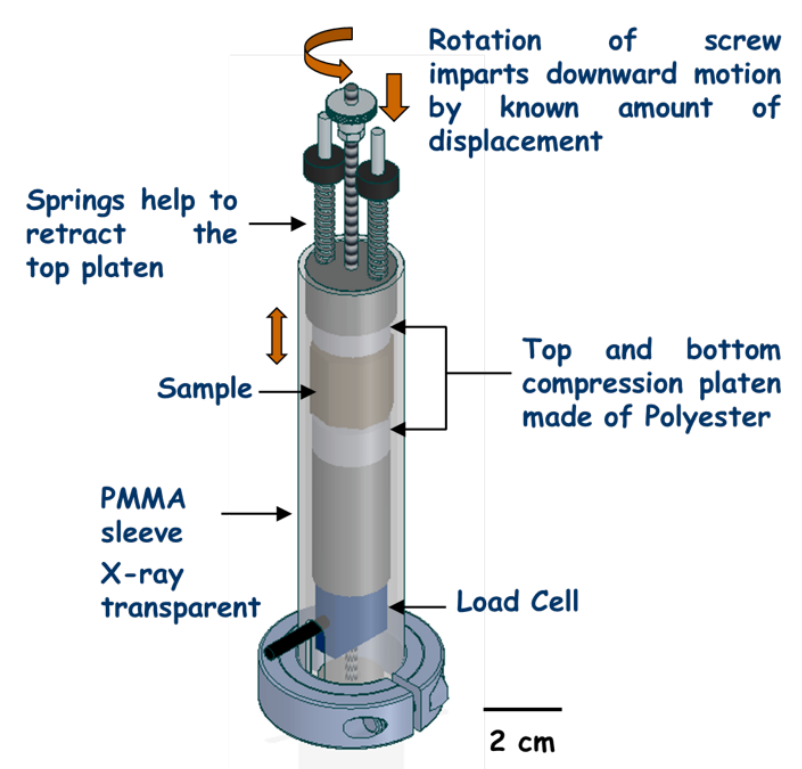

B

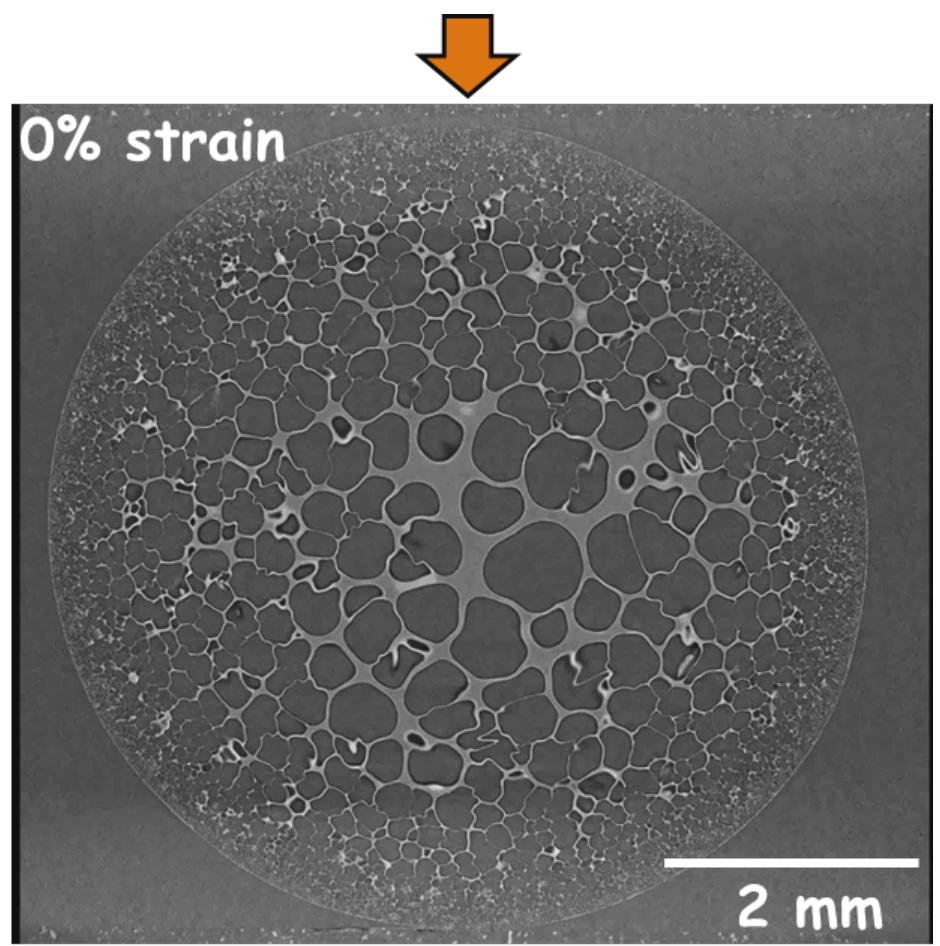

C

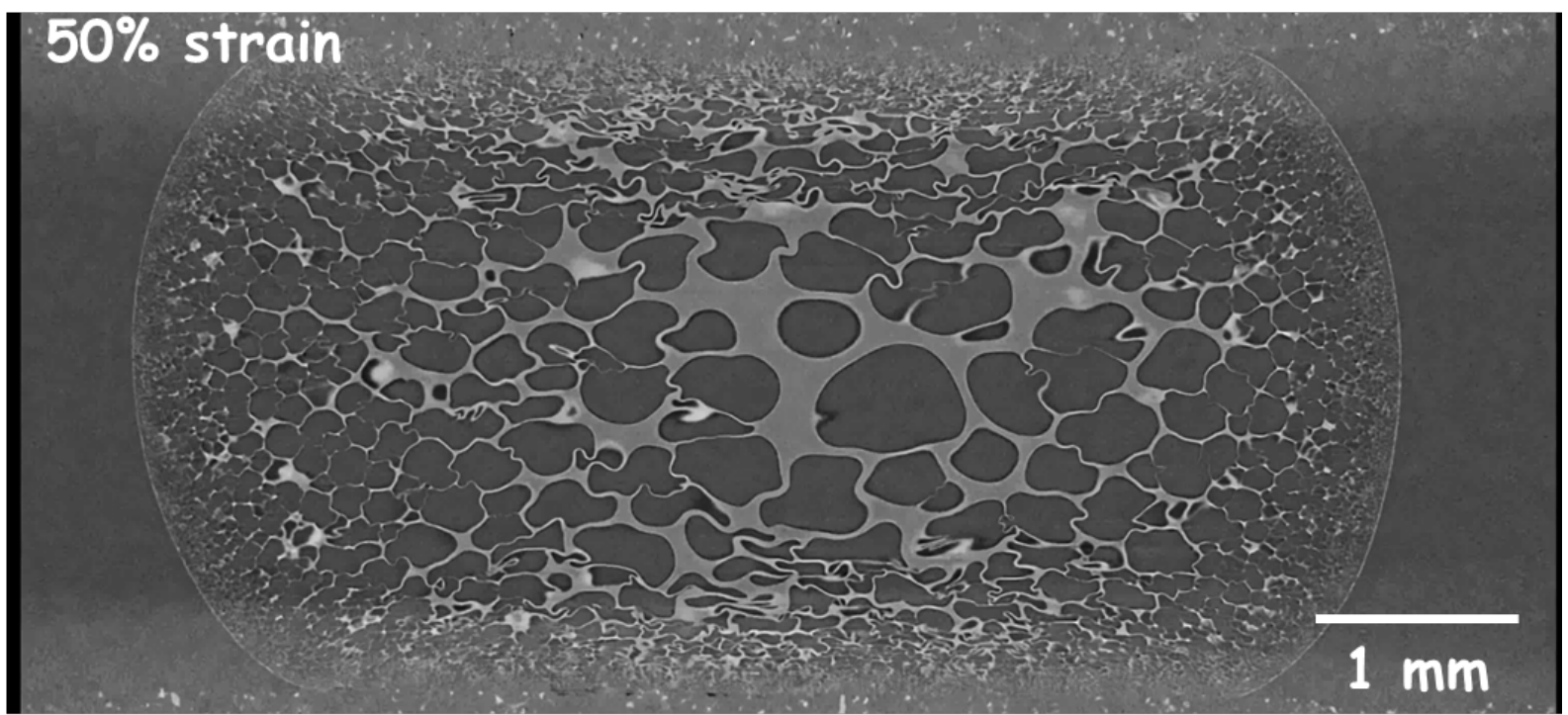

Figure 1. (A) In situ compression device for applying strain to individual eTPU beads. (B) and (C) In situ microtomography of individual beads under compression. Note the heterogeneous nature of deformation in the bead. 